\title{
AN APPLICATION OF THE SPREAD RELATION TO DIFFERENTIAL EQUATIONS
}

\author{
by GARY G. GUNDERSEN
}

(Received 16th April 1991)

\begin{abstract}
If a differential equation with meromorphic coefficients has a certain form where the growth of one of the coefficients dominates the growth of the other coefficients in a finite union of angles, then we show that this puts restrictions on the deficiencies of any meromorphic solution of the equation. We use the spread relation in the proofs. Examples are given which show that our results are sharp in several ways. Most of these examples are constructed from the quotients of solutions of $w^{\prime \prime}+G(z) w=0$ for certain polynomials $G(z)$ and from meromorphic functions which are extremal for the spread relation.
\end{abstract}

1991 Mathematics subject classification: 34A20, 30D35.

\section{Introduction}

In this paper a meromorphic function will always mean meromorphic in the whole complex plane. We will assume that the reader is familiar with the fundamental results and the standard symbols $m(r, f), N(r, f), T(r, f), \delta(c, f)$, etc., of R. Nevanlinna's theory of meromorphic functions (see [9]).

We will also use the following notation. Let $\rho(h)$ denote the order of a meromorphic function $h(z)$. For $q \geqq 1$ let $S_{1}, S_{2}, \ldots, S_{q}$ be a finite number of angles which have the form

$$
S_{i}=\left\{z: \theta_{i} \leqq \arg z \leqq \psi_{i}\right\}
$$

for $i=1,2, \ldots, q$, where $\theta_{1}<\psi_{1}<\theta_{2}<\psi_{2}<\cdots<\theta_{q}<\psi_{q}<\theta_{1}+2 \pi$. Set

$$
L=\sum_{i=1}^{q}\left(\psi_{i}-\theta_{i}\right)
$$

The main results in this paper are Theorem 1, Corollary 1, and Theorem 2 below.

Theorem 1. Suppose that we have a differential equation of the form 


$$
D(z) w^{k_{0}}\left(w^{\prime}\right)^{k_{1}} \ldots\left(w^{(m)}\right)^{k_{m}}=P\left(z, w, w^{\prime}, \ldots, w^{(v)}\right)
$$

where $D(z)$ is meromorphic, $k_{0}, k_{1}, \ldots, k_{m}$ are non-negative integers, and $P$ is a polynomial in $w, w^{\prime}, \ldots, w^{(v)}$ with meromorphic coefficients, such that

$$
\text { degree }(P) \leqq k_{0}+k_{1}+\cdots+k_{m}
$$

Suppose that there exists a finite number of angles $S_{1}, S_{2}, \ldots, S_{q}$ as in (1.1), and constants $\alpha_{i}>0, \beta_{i}>0(i=1, \ldots, q)$, such that

$$
|D(z)| \geqq \exp \left\{(1+o(1)) \alpha_{i}|z|^{\beta_{i}}\right\}
$$

as $z \rightarrow \infty$ in $S_{i}(i=1, \ldots, q)$, and such that for any coefficient $a(z)$ in $P$ we have

$$
|a(z)| \leqq \exp \left\{o(1)|z|^{\beta_{i}}\right\}
$$

as $z \rightarrow \infty$ in $S_{i}(i=1, \ldots, q)$.

Let $w=f(z)$ be a transcendental meromorphic solution of (1.3).

(i) If $m=0$, then for every value $c \neq 0$ in the extended plane we have

$$
\sin ^{-1} \sqrt{\delta(c, f) / 2} \leqq \frac{1}{4} \rho(f)(2 \pi-L)
$$

where $L$ is the number in (1.2).

(ii) If $m \geqq 1$ and $k_{m} \geqq 1$, then for every value $c$ in the extended plane except possibly for at most $q$ finite exceptional values, we have

$$
\sin ^{-1} \sqrt{\delta\left(c, f^{(m-1)}\right) / 2} \leqq \frac{1}{4} \rho(f)(2 \pi-L) .
$$

If in Theorem $1, w=f(z)$ is a rational solution of (1.3), then it is easy to see that we must have $f^{(m)} \equiv 0$. Thus we eliminate this trivial case by assuming that $f$ is transcendental in Theorem 1. Theorems 1 and 2 in [7] are special cases of Theorem 1 above.

For convenience in stating some results below, we set

$$
R(z, w)=\frac{\sum_{j=0}^{n} A_{j}(z) w^{j}}{\sum_{j=0}^{n} B_{j}(z) w^{j}}
$$

where each $A_{j}(z)$ and each $B_{j}(z)$ is meromorphic $\left(A_{n} \not \equiv 0, B_{p} \not \equiv 0\right)$, and we assume that the right side of (1.9) is completely reduced as a rational function in $w$.

We also need a definition. If $Q\left(z, w, w^{\prime}, \ldots, w^{(\mu)}\right)$ is a polynomial in $w, w^{\prime}, \ldots, w^{(\mu)}$ with meromorphic coefficients, then $Q$ is a finite sum of terms of the form

$$
H(z) w^{k_{0}}\left(w^{\prime}\right)^{k_{1}} \ldots\left(w^{(\mu)}\right)^{k_{\mu}}
$$


where $H(z)$ is meromorphic and $k_{0}, k_{1}, \ldots, k_{\mu}$ are non-negative integers. The weight of the term (1.10) is defined to be the sum

$$
k_{0}+2 k_{1}+\cdots+(\mu+1) k_{\mu},
$$

and the weight of $Q$ is defined to be the maximum weight of all of its terms (1.10).

The next result is an immediate corollary of Theorem 1 and Theorem B in Section 2.

Corollary 1. Suppose that we have the hypothesis of Theorem 1. Set

$$
\sigma=\max \{\rho(H)\}
$$

where this maximum is taken over all the coefficients $H(z)$ in equation (1.3).

Then the following two statements hold:

(i) Suppose that equation (1.3) can also be written in the form

$$
Q\left(z, w, w^{\prime}, \ldots, w^{(\mu)}\right)=R(z, w)
$$

where $Q$ is a polynomial in $w, w^{\prime}, \ldots, w^{(\mu)}$ with meromorphic coefficients, and where $R$ has the form (1.9) with $D(z) \equiv A_{n}(z)$ such that either (a) $p \geqq 1$ or $(b) p=0$ and $n>$ weight $(Q)$. Then for every value $c \neq 0$ in the extended plane we have

$$
\sin ^{-1} \sqrt{\delta(c, f) / 2} \leqq \frac{1}{4} \sigma(2 \pi-L)
$$

where $L$ is the number in (1.2).

(ii) Suppose that $m \geqq 1$ and $k_{m} \geqq 1$, and suppose that equation (1.3) can also be written in the form

$$
\left(w^{\prime}\right)^{k_{1}}\left(w^{\prime \prime}\right)^{k_{2}} \ldots\left(w^{(m)}\right)^{k_{m}}=R(z, w)
$$

where $R$ has the form (1.9) with $D(z) \equiv B_{p}(z)$ and $p \geqq 1$. Then for every value $c$ in the extended plane except possibly for at most $q$ finite exceptional values, we have

$$
\sin ^{-1} \sqrt{\delta\left(c, f^{(m-1)}\right) / 2} \leqq \frac{1}{4} \sigma(2 \pi-L) .
$$

Corollaries 1 and 2 in [7] are special cases of Corollary 1 above. We mention that if the coefficients of a differential equation in Corollary 1 (above) all have finite order, then it follows from Theorem B (in Section 2) that any meromorphic solution of the equation must also have finite order.

Theorem 1 and Corollary 1 are sharp in several ways:

(a) Examples in Section 7 will show that the four inequalities (1.7), (1.8), (1.11), and 
(1.12) are sharp in the sense that if we are given any constant $\eta$ that satisfies $0 \leqq \eta \leqq 1$, then for cases (i) and (ii) in Theorem 1 and Corollary 1, there exist equations of the form (1.3) which possess a meromorphic solution $w=f(z)$ where (1.7), (1.8), (1.11), and (1.12) reduce to an equality when $c=\infty$ and where $\delta(\infty, f)=\eta$. When $0<\eta<1$, these solutions $f(z)$ are meromorphic functions which are extremal for the spread relation.

(b) Examples in Section 8 will show that for any given integer $q \geqq 1$ in Theorem 1 or Corollary 1 , it is possible to have exactly $q$ distinct finite exceptional values in Theorem 1(ii) or Corollary 1(ii). These examples will be constructed from quotients of solutions of equations of the form $w^{\prime \prime}+G(z) w=0$ for certain polynomials $G(z)$.

(c) Examples in Section 9 will show that $c=0$ can be an exceptional value in Theorem 1(i) or Corollary 1(i).

(d) Examples in Section 10 will show that the condition (1.4) is sharp.

The next result shows that under the conditions of Theorem 1 , if we also have $\rho(f)<\infty$, then either $f$ or one of its derivatives has a finite asymptotic value in each angle $S_{i}$.

Theorem 2. Suppose that we have the hypothesis of Theorem 1 , and that $\rho(f)<\infty$. Then there exists a set $E \subset[0,2 \pi)$ that has linear measure zero such that the following two statements hold:

(i) If $m=0$ and $\phi \in S_{i}-E(i=1,2, \ldots, q)$, then

$$
f(z) \rightarrow 0
$$

as $z \rightarrow \infty$ along $\arg z=\phi$.

(ii) If $m \geqq 1$ and $k_{m} \geqq 1$, then for each $i=1,2, \ldots, q$, there exists a corresponding finite constant $c_{i}$ such that for any $\phi \in S_{i}-E$,

$$
f^{(m-1)}(z) \rightarrow c_{i}
$$

as $z \rightarrow \infty$ along $\arg z=\phi$.

In Theorem 2(ii) the constants $c_{1}, c_{2}, \ldots, c_{q}$ may or may not be distinct, while on the other hand, for each fixed $i$ the constant $c_{i}$ is independent of $\phi \in S_{i}-E$. The proof of Theorem 1 will consist of combining Theorem 2 and the spread relation (see Theorem A in Section 2).

I would like to thank Walter K. Hayman and Aimo Hinkkanen for some valuable discussions. I also thank the referee for suggestions.

\section{Preliminary results}

This section contains results that we use in the proofs of Theorem 1 , Corollary 1 , and 
Theorem 2. The first result can be deduced from the spread relation, which was conjectured by Edrei and proven by Baernstein.

Theorem A [1]. Let $h(z)$ be a transcendental meromorphic function where $\rho(h)<\infty$, and let $c$ be any value in the extended plane. Define sets of arguments $J(r, c) \subset(-\pi, \pi]$ by

$$
\begin{aligned}
J(r, c) & =\left\{\theta:\left|h\left(r e^{i \theta}\right)-c\right|<\frac{1}{r}\right\} \quad \text { if } c \neq \infty, \\
& =\left\{\theta:\left|h\left(r e^{i \theta}\right)\right|>r\right\} \quad \text { if } c=\infty .
\end{aligned}
$$

Let $\varepsilon>0$ be a given constant.

Then there exists a sequence $r_{k} \rightarrow+\infty$ such that

$$
\min \left(2 \pi, \frac{4}{\rho(h)} \sin ^{-1} \sqrt{\delta(c, h) / 2}\right) \leqq \mu\left\{J\left(r_{k}, c\right)\right\}+\varepsilon
$$

for each $r_{k}$, where $\mu\left\{J\left(r_{k}, c\right)\right\}$ denotes the linear measure of the set $J\left(r_{k}, c\right)$.

The next result is essentially due to J. Clunie, since the proof is a simple modification of the proof of Lemma 3.3 on pages 68-69 of [9].

Lemma A. Let $g(z)$ be a transcendental meromorphic solution of the equation

$$
g^{n} P=Q
$$

where $P$ and $Q$ are polynomials in $g, g^{\prime}, g^{\prime \prime}, \ldots$ with meromorphic coefficients $H_{j}(z)$. If degree $(Q) \leqq n$, then

$$
m(r, P) \leqq \sum_{j} m\left(r, H_{j}\right)+S(r, g)
$$

where $S(r, g)=o(1) T(r, g)$ as $r \rightarrow \infty$ outside a possible exceptional set of finite linear measure.

Lemma B [6]. Let h(z) be a transcendental meromorphic function of finite order $\rho$. Let $l$ and $j$ be integers that satisfy $l>j \geqq 0$ and let $\varepsilon>0$ be a given constant.

(i) There exists a set $E_{1} \subset[0,2 \pi)$ that has linear measure zero, such that if $\phi \in[0,2 \pi)-E_{1}$, then there is a constant $R_{o}=R_{o}(\phi)>0$ such that for all $z$ satisfying $\arg z=\phi$ and $|z| \geqq R_{o}$, we have

$$
\left|\frac{h^{(l)}(z)}{h^{(j)}(z)}\right| \leqq|z|^{(l-j)(\rho-1+\varepsilon)}
$$


(ii) There exists a set $E_{2} \subset(0, \infty)$ that has finite logarithmic measure, such that (2.1) holds whenever $|z| \notin E_{2} \cup[0,1]$.

The next result is a Malmquist-type theorem which comes from a theorem of Strelitz.

Theorem B. Consider a differential equation of the form

$$
Q\left(z, w, w^{\prime}, \ldots, w^{(\mu)}\right)=R(z, w)
$$

where $Q$ is a polynomial in $w, w^{\prime}, \ldots, w^{(\mu)}$ with meromorphic coefficients, and where $R$ has the form (1.9). Set

$$
\xi=\max _{j}\left\{\rho\left(H_{j}\right)\right\}
$$

where this maximum is taken over all the coefficients $H_{j}(z)$ in equation (2.2).

If (2.2) possesses a meromorphic solution $w=f(z)$ such that $\rho(f)>\xi$, then $R$ must necessarily be a polynomial in $w$ that satisfies

$$
\operatorname{degree}(R) \leqq \text { weight }(Q) \text {. }
$$

Proof. Since $\rho(f)>\xi$, it follows from a theorem of Strelitz [14] that $R$ must necessarily be a polynomial in $w$. We now make the assumption that

$$
\text { degree }(R)>\text { weight }(Q) \text {. }
$$

Let $\varepsilon>0$ be a small fixed constant. Since degree $(R)>\operatorname{degree}(Q)$ from (2.4), it follows from Lemma $A$ that

$$
m(r, f) \leqq \sum_{j} m\left(r, H_{j}\right)+S(r, f),
$$

and then from (2.3) we obtain

$$
m(r, f) \leqq O\left(r^{\xi+\varepsilon}\right)+S(r, f) .
$$

Now consider the poles of $f$. If we set $l=\operatorname{degree}(R)$, then from (2.2) and (2.4) we obtain

$$
f^{l}=P\left(z, f, f^{\prime}, \ldots, f^{(v)}\right)
$$

where $P$ is a polynomial in $f, f^{\prime}, \ldots, f^{(v)}$ with meromorphic coefficients $h_{j}(z)$ which satisfy $\rho\left(h_{j}\right) \leqq \xi$ from (2.3), and where weight $(P)<l$. Thus from (2.6) we obtain

$$
l N(r, f) \leqq \sum_{j} N\left(r, h_{j}\right)+(l-1) N(r, f),
$$

which gives

$$
N(r, f) \leqq O\left(r^{\xi+\varepsilon}\right)
$$

From (2.7) and (2.5),

$$
T(r, f) \leqq O\left(r^{\xi+\varepsilon}\right)+S(r, f)
$$


where $S(r, f)=o(1) T(r, f)$ as $r \rightarrow \infty$ outside a possible exceptional set of finite linear measure. Since $T(r, f)$ is a non-decreasing function of $r$, this implies that $[2$, p.68]

$$
T(r, f) \leqq O\left(r^{\xi+\varepsilon}\right)
$$

as $r \rightarrow \infty$ through all values of $r$. Hence $\rho(f) \leqq \xi$ which contradicts the hypothesis of Theorem B. Thus our assumption (2.4) is false, and this proves Theorem B.

We will prove the next two lemmas in Sections 3 and 4.

Lemma 1. Let $S$ be an angle $S: \theta \leqq \arg z \leqq \psi$ where $\theta<\psi$, let $\phi$ and $R_{0} \geqq 0$ be real constants where $\theta \leqq \phi \leqq \psi$, and let $\mu_{1}, \mu_{2}, \ldots$ be an infinite positive sequence that satisfies $\mu_{n} \rightarrow+\infty$ and $R_{0}<\mu_{1}<\mu_{2}<\mu_{3}<\cdots$. Let $h(z)$ be a meromorphic function that is analytic on the set $V$ defined by

$$
V=\left\{z: z \in S \text { and }|z|=\mu_{n} \text { for some } n \geqq 1\right\} \cup\left\{z: \arg z=\phi \text { and }|z| \geqq R_{o}\right\} \text {. }
$$

Let $\lambda(r)$ be a non-decreasing positive function on $R_{0} \leqq r<\infty$, and set

$$
\zeta(z)=\frac{\left|h^{(m)}(z)\right|}{\lambda(r)}
$$

where $r=|z|$ and $m \geqq 0$ is an integer. Suppose that $\zeta(z)$ is unbounded as $z \rightarrow \infty$ on $V$.

Then there exists an infinite sequence $\left\{z_{j}\right\}$ where each $z_{j} \in V$ and $z_{j} \rightarrow \infty$, such that

$$
\left|\frac{h^{(m)}\left(z_{j}\right)}{h\left(z_{j}\right)}\right| \geqq(1+o(1)) \frac{1}{\left(8\left|z_{j}\right|\right)^{m}}
$$

as $z_{j} \rightarrow \infty$, and

$$
\frac{\left|h^{(m)}\left(z_{j}\right)\right|}{\lambda\left(\left|z_{j}\right|\right)} \rightarrow \infty
$$

as $z_{j} \rightarrow \infty$.

Lemma 2. Suppose that we have the hypothesis of Theorem 1 , and that $\rho(f)<\infty$. Let $i$ be any fixed integer that satisfies $1 \leqq i \leqq q$.

Then there exists a set $E \subset[0,2 \pi)$ that has linear measure zero, an infinite positive sequence $\mu_{1}, \mu_{2}, \ldots$ that satisfies $\mu_{n} \rightarrow+\infty$ and

$$
0<\mu_{n}<\mu_{n+1}<2 \mu_{n}
$$

for all $n$, and a constant $\xi_{i}>0$ such that if $\phi \in S_{i}-E$, then

$$
\left|f^{(m)}(z)\right| \leqq \exp \left\{-(1+o(1)) \xi_{i}|z|^{\beta_{i}}\right\}
$$


as $z \rightarrow \infty$ on the set

$$
Y=\left\{z: z \in S_{i} \text { and }|z|=\mu_{n} \text { for some } n \geqq 1\right\} \cup\{z: \arg z=\phi\}
$$

\section{Proof of Lemma 1}

Since $\zeta(z)$ is unbounded as $z \rightarrow \infty$ on $V$, it follows that there exists an infinite sequence $\left\{z_{j}\right\}$ where each $z_{j} \in V$ and $z_{j} \rightarrow \infty$, such that for each $z_{j}$ we have

$$
\zeta(z) \leqq \zeta\left(z_{j}\right)
$$

for all $z \in V$ satisfying $r \leqq\left|z_{j}\right|$, and where (2.10) holds. It remains to show that (2.9) holds. Obviously (2.9) holds when $m=0$, so we assume that $m \geqq 1$.

Since $\lambda(r)$ is a non-decreasing positive function, it follows from (2.10) that

$$
h^{(m)}\left(z_{j}\right) \rightarrow \infty
$$

as $z_{j} \rightarrow \infty$, and it follows from (3.1) that

$$
\left|h^{(m)}(z)\right| \leqq\left|h^{(m)}\left(z_{j}\right)\right|
$$

for all $z \in V$ satisfying $r \leqq\left|z_{j}\right|$.

Now set $b_{0}=R_{0} e^{i \phi}$ and $z=r e^{i \eta} \in V$, and consider the equation

$$
h^{(m-1)}(z)=h^{(m-1)}\left(b_{0}\right)+\int_{\gamma} h^{(m)}(u) d u
$$

where the curve $\gamma$ first goes from the point $b_{0}$ to the point $r e^{i \phi}$ along the ray $\arg u=\phi$ and then goes from $r e^{i \phi}$ to $z=r e^{i \eta}$ along the circle $|u|=r$. Thus the curve $\gamma$ lies entirely in V.

If $r \leqq\left|z_{j}\right|$ for some $j$, then from (3.4) and (3.3) we obtain

$$
\left|h^{(m-1)}(z)\right| \leqq\left|h^{(m-1)}\left(b_{0}\right)\right|+\left(r-R_{0}+2 \pi r\right)\left|h^{(m)}\left(z_{j}\right)\right|
$$

which gives

$$
\left|h^{(m-1)}(z)\right| \leqq C_{m-1}+8 r\left|h^{(m)}\left(z_{j}\right)\right|
$$

where $C_{m-1}=\left|h^{(m-1)}\left(b_{0}\right)\right|$. Now if $m \geqq 2$ and $r \leqq\left|z_{j}\right|$, then from (3.5) we obtain

$$
\begin{aligned}
\left|h^{(m-2)}(z)\right| & \leqq h^{(m-2)}\left(b_{0}\right)\left|+\int_{\gamma}\right| h^{(m-1)}(u)|| d u \mid \\
& \leqq\left|h^{(m-2)}\left(b_{0}\right)\right|+\left(r-R_{0}+2 \pi r\right)\left(C_{m-1}+8 r\left|h^{(m)}\left(z_{j}\right)\right|\right),
\end{aligned}
$$


which gives

$$
\left|h^{(m-2)}(z)\right| \leqq C_{m-2}+8 r C_{m-1}+(8 r)^{2}\left|h^{(m)}\left(z_{j}\right)\right|
$$

where $C_{m-2}=\left|h^{(m-2)}\left(b_{0}\right)\right|$. By repeating this process as many times as necessary, we can deduce that for all $m \geqq 1$ and for any given $j$,

$$
|h(z)| \leqq \sum_{k=0}^{m-1} C_{k}(8 r)^{k}+(8 r)^{m}\left|h^{(m)}\left(z_{j}\right)\right|
$$

for all $z \in V$ which satisfy $r \leqq\left|z_{j}\right|$, where $C_{0}, C_{1}, \ldots, C_{m-1}$ are non-negative constants. By taking $z=z_{j}$ in (3.6) and noting (3.2), we obtain that (2.9) holds for all $m \geqq 1$. This completes the proof of Lemma 1.

\section{Proof of Lemma 2}

For convenience we set $S=S_{i}$ and $\rho=\rho(f)$. For equation (1.3), set $\eta=\max \{m, v\}$. Then from Lemma $B$ there exists a set $E_{1} \subset[0,2 \pi)$ that has linear measure zero and a set $E_{2} \subset(0, \infty)$ that has finite logarithmic measure, such that if $\phi \in S-E_{1}$, then there is a constant $R_{0}=R_{0}(\phi)>0$ such that for all integers $l$ and $j$ that satisfy $0 \leqq j<l \leqq \eta$, we have

$$
\left|\frac{f^{(l)}(z)}{f^{(j)}(z)}\right| \leqq|z|^{(l-j) p}
$$

for all $z$ in the set

$$
\Omega=\left\{z: \arg z=\phi \text { and }|z| \geqq R_{0}\right\} \cup\left\{z:|z| \notin E_{2} \cup[0,1]\right\} .
$$

Since $E_{2}$ has finite logarithmic measure, it follows that for any fixed real constant $t_{0}>1$ there must exist a point $x_{0}>0$ large enough, such that if $x>x_{0}$, then the interval $\left[x, t_{0} x\right]$ must contain a point that does not belong to $E_{2}$. Hence it can be deduced that we can choose an infinite positive sequence $\mu_{1}, \mu_{2}, \ldots$ such that $\mu_{n} \notin E_{2} \cup[0,1]$ for all $n$, where

$$
R_{0}<\mu_{n}<\mu_{n+1}<2 \mu_{n}
$$

for all $n$, and where $\mu_{n} \rightarrow+\infty$. Then from (4.1) and (4.2), we see that (4.1) holds for all $l$ and $j$ satisfying $0 \leqq j<l \leqq \eta$ and for all $z$ in the set

$$
V=\left\{z: z \in S \text { and }|z|=\mu_{n} \text { for some } n \geqq 1\right\} \cup\left\{z: \arg z=\phi \text { and }|z| \geqq R_{0}\right\},
$$

since $V$ is contained in $\Omega$.

For convenience we set $P(z)=P\left(z, f, f^{\prime}, \ldots, f^{(v)}\right)$ and $N=k_{0}+k_{1}+\cdots+k_{m}$ in equation 
(1.3). From consideration of (1.3) and (4.1) we can deduce that there exists a constant $\tau \geqq 0$ such that

$$
|D(z)| \cdot\left|f^{(m)}(z)\right|^{N} \leqq|z|^{\mathfrak{r}} \cdot|P(z)|
$$

for all $z \in V$. From (4.5),

$$
|D(z)| \cdot\left|\frac{f^{(m)}(z)}{f(z)}\right|^{N} \leqq \frac{\left|z^{\tau} P(z)\right|}{|f(z)|^{N}}
$$

for all $z \in V$.

We now make the assumption that

$$
\frac{\left|f^{(m)}(z)\right|}{|z|^{m \rho}}
$$

is unbounded on the set $V$. Since (4.1) holds on $V, f(z)$ is analytic on $V$. Then from the assumption and (4.4), it follows from Lemma 1 that there exists an infinite sequence $\left\{z_{j}\right\}$ where each $z_{j} \in V$ and $z_{j} \rightarrow \infty$, such that

$$
\left|\frac{f^{(m)}\left(z_{j}\right)}{f\left(z_{j}\right)}\right| \geqq(1+o(1)) \frac{1}{\left(8\left|z_{j}\right|\right)^{m}}
$$

as $z_{j} \rightarrow \infty$, and

$$
\frac{\left|f^{(m)}\left(z_{j}\right)\right|}{\left|z_{j}\right|^{\mid m \rho}} \rightarrow \infty
$$

as $z_{j} \rightarrow \infty$. From (4.8) and (4.6),

$$
\left|D\left(z_{j}\right)\right| \leqq(1+o(1)) 8^{m N}\left|z_{j}\right|^{m N+\tau} \frac{\left|P\left(z_{j}\right)\right|}{\left|f\left(z_{j}\right)\right|^{N}} .
$$

Since degree $(P) \leqq N$ from (1.4), it is easy to see that the expression

$$
\frac{P\left(z_{j}\right)}{\left(f\left(z_{j}\right)\right)^{N}}
$$

in (4.10) is made up entirely of the following three types of quantities:

(a) quantities of the form $a\left(z_{j}\right)$ where $a(z)$ is a coefficient of $P$,

(b) quantities of the form $f^{(n)}\left(z_{j}\right) / f\left(z_{j}\right)$ where $1 \leqq n \leqq \eta$, and 
(c) quantities of the form $1 / f\left(z_{j}\right)$.

In case (a) we have

$$
\left|a\left(z_{j}\right)\right| \leqq \exp \left\{o(1)\left|z_{j}\right|^{\beta_{i}}\right\}
$$

as $z_{j} \rightarrow \infty$ from (1.6). In case (b) we have

$$
\left|\frac{f^{(n)}\left(z_{j}\right)}{f\left(z_{j}\right)}\right| \leqq\left|z_{j}\right|^{n \rho}
$$

from (4.1). In case (c) we have

$$
\frac{1}{f\left(z_{j}\right)} \rightarrow 0
$$

as $z_{j} \rightarrow \infty$, because (4.13) follows directly from (4.9) when $m=0$, while if $m \geqq 1$ then (4.1) implies

$$
\frac{1}{\left|f\left(z_{j}\right)\right|} \leqq \frac{\left|z_{j}\right|^{m \rho}}{\left|f^{(m)}\left(z_{j}\right)\right|}
$$

and then (4.13) follows from (4.9).

From (4.13), (4.12), (4.11), and (1.5), we see that (4.10) yields a contradiction as $z_{j} \rightarrow \infty$. Hence our assumption that (4.7) is unbounded on the set $V$ is false.

Therefore,

$$
\left|f^{(m)}(z)\right|=O\left(|z|^{m \rho}\right)
$$

as $z \rightarrow \infty$ on $V$.

We can now use (4.14) to show that for each integer $n$ satisfying $0 \leqq n \leqq \eta$, there exists a constant $\lambda_{n}>0$ such that

$$
\left|f^{(n)}(z)\right|=O\left(|z|^{\lambda_{n}}\right)
$$

as $z \rightarrow \infty$ on $V$.

If $m \leqq n \leqq \eta$, then (4.15) will follow from (4.14) and (4.1). On the other hand, if $m \geqq 1$ and $0 \leqq n<m$, then we can obtain (4.15) by estimating in a similar manner as in the proof of Lemma 1. Specifically, set $b_{0}=R_{0} e^{i \phi}$ and $r=|z|$, and consider the equation

$$
f^{(m-1)}(z)=f^{(m-1)}\left(b_{0}\right)+\int_{\gamma} f^{(m)}(u) d u
$$

for $z \in V$, where the curve $\gamma$ first goes from the point $b_{0}$ to the point $r e^{i \phi}$ along the ray 
$\arg u=\phi$ and then from $r e^{i \phi}$ to $z$ along the circle $|u|=r$. From (4.16) and (4.14) we obtain

$$
\left|f^{(m-1)}(z)\right|=O\left(|z|^{m \rho+1}\right)
$$

as $z \rightarrow \infty$ on $V$. Thus (4.17) shows that (4.15) holds when $m \geqq 1$ and $n=m-1$. If $m \geqq 2$, then this process can be repeated with (4.17) and

$$
f^{(m-2)}(z)=f^{(m-2)}\left(b_{0}\right)+\int_{\gamma} f^{(m-1)}(u) d u .
$$

By repeating this process as many times as necessary we see that (4.15) will hold whenever $m \geqq 1$ and $0 \leqq n<m$. Hence we have shown that (4.15) holds for all $n$ satisfying $0 \leqq n \leqq \eta$.

Next we note that $N>0$ in (4.5), because if we had $N=0$, then we would have degree $(P)=0$ from (1.4), and this would imply that the differential equation (1.3) does not depend on $w$, which is absurd. Thus by using (1.5), (1.6), and (4.15) in (4.5), we obtain that

$$
\left|f^{(m)}(z)\right| \leqq \exp \left\{-(1+o(1)) \xi_{i}|z|^{\beta_{i}}\right\}
$$

as $z \rightarrow \infty$ on $V$, where $\xi_{i}=\alpha_{i} / N>0$. From (4.4) and (4.3), we see that (2.11) holds, that $\mu_{n} \rightarrow+\infty$, and that (4.18) holds as $z \rightarrow \infty$ on the set $Y$ in (2.13). By taking $E=E_{1}$, this shows that (2.12) holds, and the proof of Lemma 2 is now complete.

\section{Proof of Theorem 2}

First note that Lemma 2 holds. Let $i$ be any fixed integer that satisfies $1 \leqq i \leqq q$. Let $E \subset[0,2 \pi)$ be the set of linear measure zero in Lemma 2, and suppose that $\phi \in S_{i}-E$. Then from Lemma 2, there exists a constant $\xi_{i}>0$ such that

$$
\left|f^{(m)}(z)\right| \leqq \exp \left\{-(1+o(1)) \xi_{i}|z|^{\beta_{i}}\right\}
$$

as $z \rightarrow \infty$ along $\arg z=\phi$. If $m=0$, then (i) follows from (5.1).

Thus we now assume that $m \geqq 1$ and $k_{m} \geqq 1$. Consider the equation

$$
f^{(m-1)}\left(z_{2}\right)-f^{(m-1)}\left(z_{1}\right)=\int_{y} f^{(m)}(z) d z
$$

where $z_{1}$ and $z_{2}$ are two points of large modulus that both lie on the ray $\arg z=\phi$, and where the curve $\gamma$ goes from $z_{1}$ to $z_{2}$ along $\arg z=\phi$. From (5.2) and (5.1) it can be deduced that the quantity

$$
\left|f^{(m-1)}\left(z_{2}\right)-f^{(m-1)}\left(z_{1}\right)\right|
$$


can be made arbitrarily small by just having $\left|z_{1}\right|$ and $\left|z_{2}\right|$ large enough. It follows that there exists a constant $c_{i}$ such that

$$
f^{(m-1)}(z) \rightarrow c_{i}
$$

as $z \rightarrow \infty$ along $\arg z=\phi$.

It remains to show that the constant $c_{i}$ is independent of $\phi \in S_{i}-E$. To this end, we suppose that $\phi_{1}$ and $\phi_{2}$ satisfy $\phi_{j} \in S_{i}-E$ for $j=1,2$, and $\phi_{1} \neq \phi_{2}$. Let $\mu_{1}, \mu_{2}, \ldots$ be the sequence in Lemma 2 , and we note that

$$
0<\mu_{n}<\mu_{n+1}<2 \mu_{n}
$$

holds for all $n$, from (2.11). Set

$$
Y_{1}=\left\{z: z \in S_{i} \text { and }|z|=\mu_{n} \text { for some } n \geqq 1\right\} \cup\left\{z: \arg z=\phi_{1}\right\}
$$

and

$$
Y_{2}=\left\{z: z \in S_{i} \text { and }|z|=\mu_{n} \text { for some } n \geqq 1\right\} \cup\left\{z: \arg z=\phi_{2}\right\}
$$

Consider the equation

$$
f^{(m-1)}\left(b_{2}\right)-f^{(m-1)}\left(b_{1}\right)=\int_{\Gamma} f^{(m)}(z) d z
$$

where $b_{1}$ and $b_{2}$ are two points of large modulus such that $\arg b_{1}=\phi_{1}$ and $\arg b_{2}=\phi_{2}$, and where the curve $\Gamma$ lies entirely in the set $Y_{1} \cup Y_{2}$ and is chosen so that the length of $\Gamma$ is as small as possible. Since (5.1) holds as $z \rightarrow \infty$ on $Y_{1} \cup Y_{2}$ from Lemma 2, it can be deduced from (5.5) and (5.4) that the quantity

$$
\left|f^{(m-1)}\left(b_{2}\right)-f^{(m-1)}\left(b_{1}\right)\right|
$$

can be made arbitrarily small by just having $\left|b_{1}\right|$ and $\left|b_{2}\right|$ large enough. It follows that the constant $c_{i}$ in (5.3) must be the same constant for all $\phi \in S_{i}-E$. This proves (ii) and the proof of Theorem 2 is complete.

\section{Proof of Theorem 1}

From (1.1) and (1.2) we have $0<L<2 \pi$. Thus (1.7) and (1.8) are obviously true if $\rho(f)=\infty$. Hence we assume that $\rho(f)<\infty$.

Let $E \subset[0,2 \pi)$ be the set of linear measure zero in Theorem 2 . Let $\varepsilon>0$ be a given small constant.

First suppose that $m=0$. Since $E$ has linear measure zero, it follows from (1.13), Theorem $A$, and (1.2), that for any value $c \neq 0$ in the extended plane, we have 


$$
\min \left(2 \pi, \frac{4}{\rho(f)} \sin ^{-1} \sqrt{\delta(c, f) / 2}\right) \leqq 2 \pi-L+\varepsilon
$$

By letting $\varepsilon \rightarrow 0$ we obtain (1.7). This proves (i)

Now suppose that $m \geqq 1$ and $k_{m} \geqq 1$. Let $c_{1}, c_{2}, \ldots, c_{q}$ be the finite constants in Theorem 2(ii). Since $E$ has linear measure zero, it follows from (1.14), Theorem A, and (1.2), that for any value $c \neq c_{i}(i=1,2, \ldots, q)$ in the extended plane, we have

$$
\min \left(2 \pi, \frac{4}{\rho(f)} \sin ^{-1} \sqrt{\delta\left(c, f^{(m-1)}\right) / 2}\right) \leqq 2 \pi-L+\varepsilon
$$

Letting $\varepsilon \rightarrow 0$ gives (1.8). This proves (ii).

The proof of Theorem 1 is complete.

\section{The inequalities are sharp}

We will first illustrate that the four inequalities (1.7), (1.8), (1.11), and (1.12) in Theorem 1 and Corollary 1 are sharp for any given numerical value of the deficiency $\delta(c, f)$. Speifically, we will show that if $\eta$ is any given constant that satisfies $0 \leqq \eta \leqq 1$, then for cases (i) and (ii) in Theorem 1 and Corollary 1, there exist equations of the form (1.3) which possess a meromorphic solution $w=f(z)$ such that the corresponding inequality reduces to an equality when $c=\infty$ and where $\delta(\infty, f)=\eta$.

Suppose first that $0<\eta<1$. Let $\tau \geqq 2$ be an integer and let $\rho$ be any real constant that satisfies $\tau-1 / 2<\rho<\tau$. Let $f(z)$ be the meromorphic function $f(z)$ that is constructed in Section 7 of [7], i.e., $f(z)$ is defined by (7.5) on page 73 of [7]. For the discussion below concerning this particular function $f(z)$, we refer the reader to Section 7 in [7].

We have

$$
\begin{gathered}
\rho(f)=\rho, \\
\delta(\infty, f)=\eta,
\end{gathered}
$$

and $f(z)$ is an extremal function for the spread relation. Now set

$$
\xi=\frac{2 \sin ^{-1} \sqrt{\eta / 2}}{\rho} \text { and } \beta=\tau-1 / 2,
$$

and let $\varepsilon>0$ be a given small constant. Then there exists a constant $C=C(\varepsilon)>0$ and a finite number of angles $S_{1}, S_{2}, \ldots, S_{q}$ as in (1.1) such that the number $L$ in (1.2) satisfies

$$
2 \pi-2 \xi-\varepsilon \leqq L<2 \pi-2 \xi
$$

and 


$$
|f(z)| \leqq \exp \left\{-(1+o(1)) C|z|^{\beta}\right\}
$$

as $z \rightarrow \infty$ in $S_{i}(i=1,2, \ldots, q)$.

In $N \geqq 0$ is any fixed integer, then by applying the Cauchy integral formula to (7.2), we can deduce that for all $j$ satisfying $0 \leqq j \leqq N$,

$$
\left|f^{(j)}(z)\right| \leqq \exp \left\{-(1+o(1)) C|z|^{\beta}\right\}
$$

as $z \rightarrow \infty$ in $S_{i}(i=1, \ldots, q)$. We note that we may have to make the angles $S_{1}, \ldots, S_{q}$ slightly smaller in order to obtain (7.3) from (7.2), but this will not affect anything since the condition (7.1) will still be satisfied.

By using the estimates in (7.3) it is easy to construct examples of differential equations for which the inequalities (1.7), (1.8), (1.11), and (1.12) will reduce to equalities. For example, in each of the following seven equations, let the meromorphic coefficient $D_{j}(z)$ $(j=1,2, \ldots, 7)$ be defined by the equation:

1. $D_{1}(z) f^{2}\left(f^{\prime}\right)^{3}=f f^{\prime \prime}-\left(f^{\prime \prime \prime}\right)^{5}+1$.

2. $D_{2}(z)\left(f^{\prime}\right)^{6}=\left(f^{(8)}\right)^{2}+e^{z} f^{\prime \prime} f^{\prime \prime \prime}-f f^{\prime}+\sin z$.

3. $D_{3}(z) f^{3}=e^{z} f^{\prime}\left(f^{\prime \prime}\right)^{2}-z^{3} f^{(5)}+1$.

4. $D_{4}(z) f^{4}=(\tan z) f^{\prime}+(\sin z)\left(f^{\prime}\right)^{3}-e^{z}$.

5. $D_{5}(z) f^{3}\left(f^{\prime}\right)^{7}=f-\left(f^{\prime}\right)^{7}+e^{z} f^{2}+1$.

6. $D_{6}(z) f^{8}=f+f^{3}+\left(1+f+f^{2}\right)\left\{1+\left(f^{\prime \prime \prime}\right)^{4}+\left(f^{\prime}\right)^{2}\left(f^{(6)}\right)^{3}+f^{\prime \prime}\right\}$.

7. $D_{7}(z) f^{9}=f f^{\prime \prime \prime}+\left(f^{\prime \prime}\right)^{2}-e^{z} f^{7}+f+f^{(5)}+1$.

We can use (7.3) and $\beta>1$ to deduce that for each $j=1, \ldots, 7$, there exists a constant $\alpha=\alpha(j, \varepsilon)>0$ such that

$$
\left|D_{j}(z)\right| \geqq \exp \left\{(1+o(1)) \alpha|z|^{\beta}\right\}
$$

as $z \rightarrow \infty$ in each $S_{i}(i=1,2, \ldots, q)$. Thus each of these seven equations gives an example for Theorem 1, while equations 5, 6, and 7 give examples for Corollary 1 also. For each of the seven examples, either the inequality (1.7) or the inequality (1.8) when $c=\infty$ is approaching an equality ( $\rho \xi / 2$ on both sides) as $\varepsilon \rightarrow 0$, and we have $\delta(\infty, f)=\eta$. In equations 5,6 , and 7 we have $\rho(f)=\rho\left(D_{j}\right)$ for $j=5,6,7$, because $\rho(f) \leqq \rho\left(D_{j}\right)$ from Theorem B, while $\rho(f) \geqq \rho\left(D_{j}\right)$ is obvious. Thus for each of the equations 5,6 , and 7 , either the inequality (1.11) or the inequality (1.12) when $c=\infty$ approaches an equality $(\rho \xi / 2)$ as $\varepsilon \rightarrow 0$, and we have $\delta(\infty, f)=\eta$. Hence these examples show that each of the four inequalities (1.7), (1.8), (1.11), and (1.12) is sharp for any given numerical value of $\eta=\delta(c, f)$ satisfying $0<\eta<1$.

By using elementary functions it is very easy to generate examples which show that these four inequalities are sharp when either $\eta=0$ or $\eta=1$. As one example, for the case 
when $\eta=\delta(\infty, f)=1$ in (ii) of Theorem 1 , we have that $w=e^{-z}$ satisfies $e^{z} w\left(w^{\prime}\right)^{2}=$ $w-e^{-z} w^{\prime \prime \prime}-e^{-z}$. See also [7].

\section{8. $q$ exceptional values are possible}

We will next construct examples which show that for any given integer $q \geqq 1$ in Theorem 1 or Corollary 1 , it is possible to have exactly $q$ distinct finite exceptional values in Theorem 1(ii) or Corollary 1(ii).

First we suppose that $q \geqq 2$. Let $G(z) \neq 0$ be a polynomial of degree $q-2$. It is well known [3] that any solution $w \neq 0$ of

$$
w^{\prime \prime}+G(z) w=0
$$

is entire with $\rho(w)=q / 2$. For the discussion below concerning equation (8.1) we refer the reader to $[4,5,10,11,12,13]$.

Set $\beta=q / 2$, and let $\varepsilon>0$ be a given small constant. Then there exist $q$ angles $S_{1}, S_{2}, \ldots, S_{q}$ as in (1.1), where

$$
L=2 \pi-\varepsilon
$$

in (1.2), and there exists a constant $\xi=\xi(\varepsilon)>0$ such that for any solution $w \neq 0$ of (8.1) and any angle $S_{i}$ we must have either

$$
|w(z)| \geqq \exp \left\{(1+o(1)) \xi|z|^{\beta}\right\}
$$

as $z \rightarrow \infty$ in $S_{i}$, or

$$
|w(z)| \leqq \exp \left\{-(1+o(1)) \xi|z|^{\beta}\right\}
$$

as $z \rightarrow \infty$ in $S_{i}$. (The angles $S_{1}, \ldots, S_{q}$ can be chosen so that they each have the same angle measure.) In most cases (8.3) will hold.

On the other hand, Hellerstein and Rossi [10] have shown that there exists a polynomial $G_{q-2}(z)$ of degree $q-2$ such that the equation

$$
w^{\prime \prime}+G_{q-2}(z) w=0
$$

will possess $q$ pairwise linearly independent solutions $w_{1}, w_{2}, \ldots, w_{q}$ such that for each $j=1,2, \ldots, q, w_{j}(z)$ satisfies (8.4) as $z \rightarrow \infty$ in $S_{j}$, and $w_{j}(z)$ satisfies (8.3) as $z \rightarrow \infty$ in $S_{i}$ for all $i \neq j$. Concrete examples of this type are $G_{q-2}(z)=-z^{q-2}$ (see [8]).

It is well known that there exists two linearly independent solutions $w=f_{1}(z)$ and $w=f_{2}(z)$ of (8.5) which both satisfy $(8.3)$ as $z \rightarrow \infty$ in each $S_{i}(i=1,2, \ldots, q)$. Since the solutions $w_{1}, w_{2}, \ldots, w_{q}$ are pairwise linearly independent, it follows that there exist distinct non-zero constants $c_{1}, c_{2}, \ldots, c_{q}$ such that 


$$
f_{1}-c_{i} f_{2}=a_{i} w_{i}
$$

for $i=1,2, \ldots, q$, where $a_{1}, a_{2}, \ldots, a_{q}$ are non-zero constants.

Now set

$$
f=\frac{f_{1}}{f_{2}} .
$$

From the growth properties of $f_{1}, f_{2}$ and $w_{1}, w_{2}, \ldots, w_{q}$ in each angle $S_{i}$, we see from (8.6) that

$$
f(z) \rightarrow c_{i}
$$

as $z \rightarrow \infty$ in $S_{i}(i=1,2, \ldots, q)$. It is well known that

$$
\delta\left(c_{i}, f\right)=\frac{2}{q}
$$

for each $i=1,2, \ldots, q$.

Since $f^{\prime}=b /\left(f_{2}\right)^{2}$ where $b \neq 0$ is a constant from (8.7) and Abel's identity, and since $f_{2}$ satisfies (8.3) in each $S_{i}$, it follows from the Cauchy integral formula that for any given integer $N \geqq 1$ we have for all $j$ satisfying $1 \leqq j \leqq N$,

$$
\left|f^{(j)}(z)\right| \leqq \exp \left\{-(1+o(1)) 2 \xi|z|^{\beta}\right\}
$$

as $z \rightarrow \infty$ in each $S_{i}(i=1,2, \ldots, q)$. (The angles $S_{i}$ may have to be made slightly smaller to obtain (8.10), but this does not affect anything.)

From the estimates in (8.10), it will be easy to construct the kind of examples that we desire. For example, in each of the following four equations, let the meromorphic coefficient $D_{j}(z)(j=1,2,3,4)$ be defined by the equation (where $M>0$ is a very large constant):

1. $D_{1}(z) f^{2} f^{\prime}=1+\left(f^{(6)}\right)^{2}+\left(f^{\prime \prime}\right)^{3}$.

2. $D_{2}(z)\left(f^{\prime}\right)^{4}=M+f^{3}+f^{\prime \prime} f^{(8)}$.

3. $D_{3}(z) f^{3}\left(f^{\prime}\right)^{2}=M+f^{2}-f\left(f^{\prime}\right)^{2}-f^{5}$.

4. $D_{4}(z) f^{6}\left(f^{\prime}\right)^{7}=M-f^{3}-\left(f^{\prime}\right)^{7}+f^{4}+f^{8}$.

We can use (8.10), (8.8), and $c_{i} \neq 0$ for $i=1, \ldots, q$, to deduce that for each $j=1,2,3,4$, there exists a constant $\alpha=\alpha(j, \varepsilon)>0$ such that

$$
\left|D_{j}(z)\right| \geqq \exp \left\{(1+o(1)) \alpha|z|^{\beta}\right\}
$$

as $z \rightarrow \infty$ in each $S_{i}(i=1,2, \ldots, q)$. Thus each of these equations gives an example of 
Theorem 1(ii), while equations 3 and 4 give examples for Corollary 1 (ii) also. From (8.2) and $\rho(f)<\infty$, we see that for these four equations the right side of the inequality (1.8) approaches 0 as $\varepsilon \rightarrow 0$. Hence from (8.9), we see that (1.8) does not hold for the $q$ distinct finite values $c_{1}, c_{2}, \ldots, c_{q}$. Similarly, for equations 3 and 4 above, the inequality (1.12) does not hold for the $q$ values $c_{1}, c_{2}, \ldots, c_{q}$. This follows from (8.9) and the observation that the right side of (1.12) approaches 0 as $\varepsilon \rightarrow 0$ (since $\rho\left(D_{3}\right)<\infty$ and $\rho\left(D_{4}\right)<\infty$ are immediate from equations 3 and 4).

Hence these examples show that for any given integer $q \geqq 2$ in Theorem 1 or Corollary 1 , it is possible to have exactly $q$ distinct finite exceptional values in Theorem 1(ii) or Corollary 1(ii).

It is also possible to have $q=1$ in Theorem 1 or Corollary 1 and to have exactly $q=1$ finite exceptional value in Theorem 1(ii) or Corollary 1(ii). For example, $w=1+\sec \sqrt{z}$ satisfies $D(z) w w^{\prime}=w-w^{\prime}$ where

$$
D(z)=\frac{2 \sqrt{z} \cot \sqrt{z}(1+\cos \sqrt{z})-1}{1+\sec \sqrt{z}},
$$

and the exceptional value is $c=1$.

\section{9. $c=0$ can be exceptional}

The function $w=\sec z$ is a solution of each of the following three equations:

1. $(\cos z) w^{3}=w w^{\prime \prime}-(\sec z)^{4}+(\cot z)^{2}\left(w^{\prime}\right)^{2}-(\tan z)^{2} w^{2}$,

2. $(\sin z) w^{3}=w^{\prime}+w w^{\prime}-(\tan z) w$,

3. $(\cos z) w^{5}=w^{\prime \prime}-(\tan z)^{2} w-w^{3}+w^{4}$.

These three examples show that $c=0$ can be an exceptional value in Theorem 1(i) or Corollary 1(i). Example 1 illustrates this property about Theorem 1(i), Example 2 illustrates this property about case (a) in Corollary 1(i), and Example 3 illustrates this property about case (b) in Corollary 1(i).

\section{Condition (1.4) is sharp}

The function $w=\sin z$ is a solution of each of the following four equations:

1. $(\cos z) w^{7}=(\cot z)\left(w^{\prime \prime}\right)^{8}$,

2. $(\sin z) w^{2}\left(w^{\prime}\right)^{4}=-(\tan z)^{3}\left(w^{\prime \prime \prime}\right)^{7}$,

3. $(\sin z) w^{3}=(\csc z) w+(\tan z) w w^{\prime}\left(w^{(4)}\right)^{2}-1$,

4. $(\sin z) w^{2}\left(w^{\prime}\right)^{5}=(\csc z)(\cot z)^{5} w^{6}+(\cot z)^{5} w^{8}-\left(w^{\prime}\right)^{5}$.

These four examples show that the condition (1.4) is sharp with respect to all the possible cases in Theorem 1 and Corollary 1. All four examples illustrate this property 
about Theorem 1, while examples 3 and 4 illustrate this property about Corollary 1 also. Specifically, for each of the four examples, the conclusion in the respective result (i.e., in Theorem 1 or Corollary 1 or both) does not hold when $c=\infty$ while at the same time all of the hypothesis in the respective result holds except for (1.4) where we have $\operatorname{deg}(P)=k_{0}+k_{1}+\cdots+k_{m}+1$ in each of the four examples. We observe that if $\operatorname{deg}(P)>$ $k_{0}+k_{1}+\cdots+k_{m}$, then case (b) in Corollary 1(i) is not possible.

\section{REFERENCES}

1. A. Baernstein II, Proof of Edrei's spread conjecture, Proc. London Math. Soc. (3) 26 (1973), 418-434.

2. S. BANK, A general theorem concerning the growth of solutions of first-order algebraic differential equations, Compositio Math. 25 (1972), 61-70.

3. S. Bank and I. Laine, On the oscillation theory of $f^{\prime \prime}+A f=0$ where $A$ is entire, Trans. Amer. Math. Soc. 273 (1982), 351-363.

4. W. H. J. Fuchs, Topics in Nevanlinna theory, in Proceedings of the NRL Conference on Classical Function Theory (F. Gross, Editor, Naval Research Laboratory, Washington, D.C., 1970), 1-32.

5. G. Gundersen, On the real zeros of solutions of $f^{\prime \prime}+A(z) f=0$ where $A(z)$ is entire, $A n n$. Acad. Sci. Fenn. Ser. A I Math. 11 (1986), 275-294.

6. G. GUNDERSEN, Estimates for the logarithmic derivative of a meromorphic function, plus similar estimates, J. London Math. Soc. (2) 37 (1988), 88-104.

7. G. GUNDERSEN, The deficiencies of meromorphic solutions of certain algebraic differential equations, Results in Math. 16 (1989), 54-76.

8. G. Gundersen and E. Steinbart, A generalization of the Airy integral for $f^{\prime \prime}-z^{n} f=0$, Trans. Amer. Math. Soc., to appear.

9. W. K. Hayman, Meromorphic Functions (Clarendon Pres, Oxford, 1964).

10. S. Hellerstein and J. Rossi, Zeros of meromorphic solutions of second order linear differential equations, Math. Z. 192 (1986), 603-612.

11. E. Hille, Lectures on Ordinary Differential Equations (Addison-Wesley, Reading, Massachusetts, 1969).

12. F. Nevanuinna, Über eine Klasse meromorpher Funktionen (Septième Congress Math. Scand., Oslo, 1929).

13. R. Nevanuinna, Über Riemannsche Flächen mit endlich vielen Windungspunkten, Acta Math. 58 (1932), 295-373.

14. Sh. Strelitz, On meromorphic solutions of algebraic differential equations, Trans. Amer. Math. Soc. 258 (1980), 431-440.

Department of Mathematics

UNIVERSITY OF NeW ORLEANS

New Orleans, Louisiana 70148

U.S.A. 